\title{
A Case of Isolated Left Ventricular Noncompaction with Basal ECG-Tracing Strongly Suggestive for Type-2 Brugada Syndrome
}

\author{
Maria Banci, ${ }^{1}$ Roberta Martinoli, ${ }^{2}$ Alessandro Dofcaci, ${ }^{1}$ Stefano Piccirilli, ${ }^{3}$ \\ Federica Papetti, ${ }^{1,4}$ Ilaria Sansoni, ${ }^{5}$ and Patrizia Saccucci ${ }^{2}$ \\ ${ }^{1}$ Department of Cardiology, Valmontone Hospital, 00038 Valmontone, Italy \\ ${ }^{2}$ Department of Biopathology and Imaging Diagnostics, University of Rome "Tor Vergata", Via Montpellier 1, 00133 Rome, Italy \\ ${ }^{3}$ Department of Cardiology, University of Rome "Tor Vergata", 00133 Rome, Italy \\ ${ }^{4}$ Department of Heart and Great Vessels "A. Reale", Sapienza University of Rome, 00185 Rome, Italy \\ ${ }^{5}$ Department of Imaging Diagnostics, Campus Bio-Medico University, 00128 Rome, Italy
}

Correspondence should be addressed to Patrizia Saccucci, saccucci@med.uniroma2.it

Received 7 August 2010; Accepted 30 September 2010

Academic Editor: Gregory Giamouzis

Copyright () 2011 Maria Banci et al. This is an open access article distributed under the Creative Commons Attribution License, which permits unrestricted use, distribution, and reproduction in any medium, provided the original work is properly cited.

Isolated left ventricular noncompaction (ILVNC) is a cardiomyopathy caused by intrauterine arrest of compaction of the myocardial fibres and meshwork, an important process in myocardial development. ILVNC is clinically accompanied by depressed ventricular function, arrhythmias, and systemic embolization. We reported a case of ILVNC with basal ECG-tracing strongly suggestive for type-2 Brugada syndrome (BrS). Up to now, this is the first report investigating the association between ILVNC and this particular ECG pattern.

\section{Introduction}

Isolated left ventricular noncompaction (ILVNC) is a relatively newly defined disorder of the endomyocardium characterized by prominent ventricular trabeculations and deep intertrabecular recesses [1]. Clinical manifestations include a depressed left ventricular function, ventricular arrhythmia, and systemic embolization [2, 3]. ILVNC has been reported to be associated with several electrocardiographic changes such as ST depression and flat or negative T waves, bundle branch block, and Wolff-Parkinson-White (WPW) syndrome [2-4]. To the best of our knowledge, Brugada syndrome $(\mathrm{BrS})$ has not been reported on previously. We described a case of ILVNC with basal ECG tracing strongly suggestive for type- $2 \mathrm{BrS}$.

\section{Case Report}

We reported the case of a 59-year-old man with basal ECG tracing strongly suggestive for type-2 BrS Figure 1(c). He had virtually no cardiac complaint or any medication before. On physical examination, his heart rate was 70 beats $/ \mathrm{min}$, blood pressure was $130 / 80 \mathrm{mmHg}$, and body temperature was $36.1^{\circ} \mathrm{C}$. Cardiopulmonary findings were normal. Haematological and biochemical tests were within normal limits.

Patient underwent a transthoracic echocardiogram (TTE) that excluded arrhythmogenic right ventricular dysplasia, septal ischemia, ventricular aneurysm, and fast repolarization and showed noncompaction areas in both ventricles. This exam revealed the existence of two layers of myocardium - a thin, compacted, epicardial layer and a thick, noncompacted endocardial zone. The inner layer consisted of multiple myocardial trabeculations and deep intratrabecular recesses communicating with the left ventricular cavity Figures 1 (a) and 1(b). The ratio of systolic thickness of noncompacted to compacted myocardium layers was above $2.0(\mathrm{~N} / \mathrm{C}>2.0)$. In the colour-Doppler the deep intertrabecular recesses were filled with blood from the ventricular cavity. This finding was confirmed by nuclear magnetic resonance (NMR). 


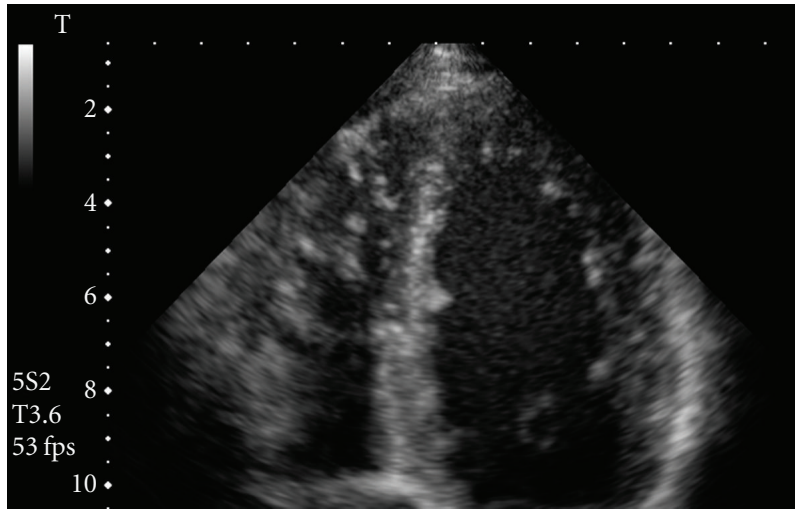

(a)

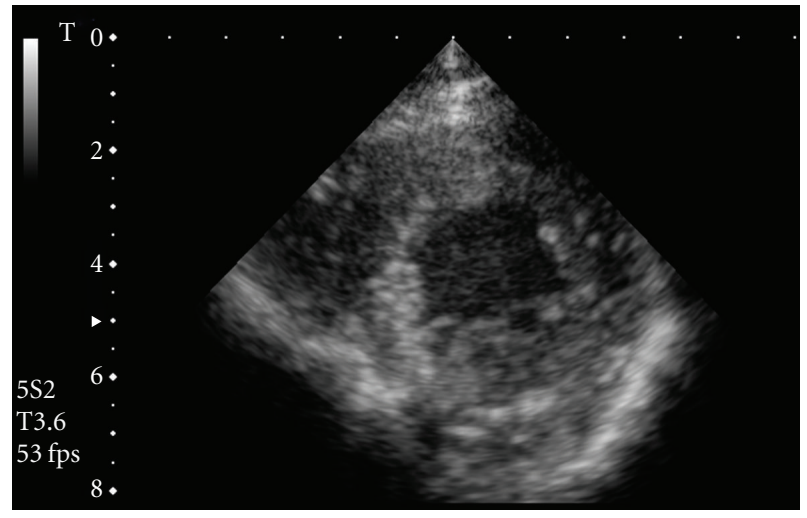

(b)

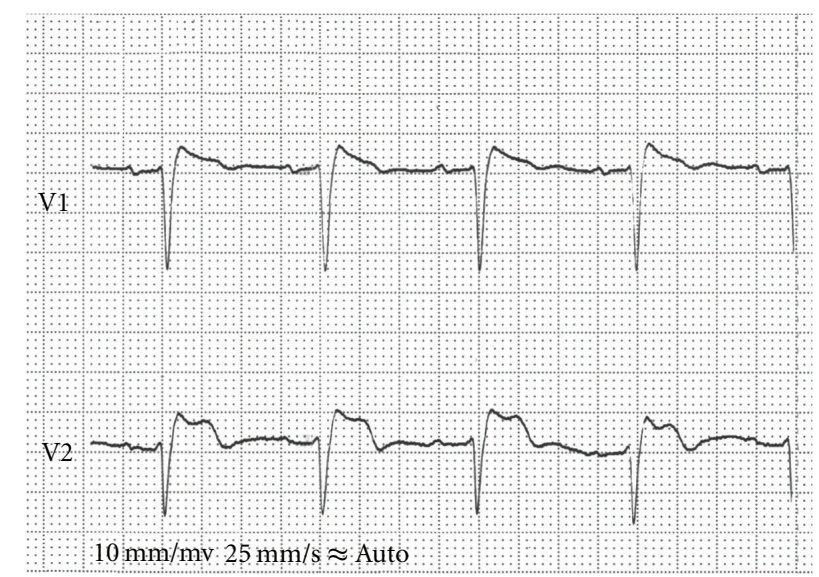

(c)

Figure 1: (a) Four-chamber view showing left and right ventricular noncompaction. The inner noncompacted layer is thick and hyperechogenic, and the outer is compacted and echolucent. This appearance is more markedly visible on the left ventricle's lateral wall and apex and on the right ventricle's free wall and apex. (b) Left ventricular short axis view showing noncompacted areas in the apex. (c) Type-2 ECG pattern with ST-segment elevation followed by a positive T wave in V1 and V2 consistent with Brugada syndrome.

The Holter ECG did not reveal any ipercinetic arrhythmias but confirmed ST-segment elevation in the right precordial leads (V1 and V2). Treadmill stress test according to the Bruce protocol was normally sustained for BP and ECG ischemic findings. During the recovery phase the saddlebacktype ST-segment elevation in V1 and V2 changed into a coved-type ECG. The ventricular tachycardia elicited by the flecainide test was hemodynamically stable and normalized without medication.

Based on the dynamic ECG changes (conversion from saddleback- to coved-type ECG) and exercise-induced ventricular tachycardia showing a left bundle branch block pattern, a diagnosis of asymptomatic Brugada syndrome was made.

The patient was sent to an arrhythmologic center for the electrophysiological study.

\section{Discussion}

ILVNC is a rare congenital cardiomyopathy characterized by excessively prominent trabecular meshwork and deep intratrabecular recesses within the left ventricle, sometimes also affecting the right ventricle and interventricular septum $[4,5]$. The predominant clinical features are heart failure, arrhythmias, embolic events, and sudden cardiac death (SCD) [4]. By definition, ILVNC occurs in the absence of other structural heart diseases. The gold standard for the ILVNC diagnosis is the echocardiographic examination. Because of an increasing awareness and interest in this disorder, and advances in echocardiographic imaging, reported cases have increased.

Although the etiology of ILVNC has not been fully investigated, this disorder is thought to result from an arrest in endomyocardial morphogenesis during the embryonic period [1]. During early embryonic life, the heart is a loose interwoven meshwork of muscle fibers. The developing myocardium gradually condenses, and the large spaces within the trabecular meshwork flatten or disappear [6].

The ECG of the previously reported patients with ILVNC showed ST depression and flat or negative $\mathrm{T}$ waves, bundle branch block, WPW syndrome, and various patterns of arrhythmia [2-4]. A high incidence of WPW syndrome 
was found in children [4]. A basal ECG tracing strongly suggestive for type-2 BrS accompanied by ILVNC has not yet been reported.

Brugada syndrome (BrS; OMIM 601144) is an autosomal dominant arrhythmogenic pathology characterized by syncope and SCD. This pathology, defined by some authors as "Idiopathic ventricular fibrillation," is actually understood as a channelopathy probably consecutive to mutations on SCN5A gene (3p21-23) encoding the cardiac sodium channel's $\alpha$-subunit [7].

BrS manifests with syncope and cardiac arrest, typically occurring in the third and fourth decade of life, and usually at rest or during sleep. This eventuality called for an aggressive therapeutic strategy in all patients with $\mathrm{BrS}$ and, since no pharmacological treatment of proven efficacy still is available, it led to implantable cardioverter defibrillator (ICD) implants.

According to a recent consensus conference [8], $\mathrm{BrS}$ is diagnosed in presence of the following diagnostic criteria.

(1) Type-1 ECG pattern with ST-segment elevation in more than one right precordial lead, followed by a negative $\mathrm{T}$ wave plus one of the following conditions documented ventricular fibrillation; self-terminating polymorphic ventricular tachycardia; a family history of SCD ( $<45$ years); coved-type ECGs in family members; electrophysiological inducibility; syncope or nocturnal agonal respiration.

(2) Appearance of type-2 ECG pattern with ST-segment elevation in more than one right precordial lead upon challenge with a sodium channel blockers (Flecainide or Ajmaline), followed by a positive or biphasic $\mathrm{T}$ wave that results in a saddleback configuration.

(3) Appearance of type-3 ECG pattern characterized by ST-segment elevation $(\leq 1 \mathrm{~mm})$ in more than one lead either with a coved-type or a saddleback morphology, with conversion to type 1 after challenge with a sodium channel blocker.

In their initial report on eight patients, Brugada et al. emphasized the lack of structural cardiac abnormalities [9]. However, there is probably an overlap between Brugada syndrome and other nosographic entities. It is reported in the literature the association between the typical V1V3 segment elevation and right bundle branch block in some patients with arrhythmogenic right ventricular cardiomyopathy (ARVC; OMIM 107970) [10, 11]. Molecular genetic studies identified five allelic variants cosegregating with dilated cardiomyopathy (DCM) $[12,13]$. Some authors reported structural degeneration (fibrosis) and apoptosis during the analysis of myocardial biopsies of patients with clinical diagnosis of Brugada syndrome and an SCN5A mutation [14]. Taken as a whole, these studies suggest that at least some SCN5A mutations manifest as both excitability and structural derangement.

In the presented case, there was no conclusive proof of the aetiology and the coexistence of the Brugada-type electrocardiographic pattern and ILVNC might be coincidental. However, the case is consistent with the consideration that up to now none of the autopsies in Brugada patients turned out to present a normal heart.

On a clinical basis, the possible coexistence of the Brugada syndrome with structural myocardial alterations should always be considered and it advocates the need of careful echo and NMR studies on all BrS patients.

\section{References}

[1] R. Jenni, E. Oechslin, J. Schneider, C. Attenhofer Jost, and P. A. Kaufmann, "Echocardiographic and pathoanatomical characteristics of isolated left ventricular non-compaction: a step towards classification as a distinct cardiomyopathy," Heart, vol. 86, no. 6, pp. 666-671, 2001.

[2] R. H. Pignatelli, C. J. McMahon, W. J. Dreyer et al., "Clinical characterization of left ventricular noncompaction in children: a relatively common form of cardiomyopathy," Circulation, vol. 108, no. 21, pp. 2672-2678, 2003.

[3] M. Ritter, E. Oechslin, G. Sütsch, C. Attenhofer, J. Schneider, and R. Jenni, "Isolated noncompaction of the myocardium in adults," Mayo Clinic Proceedings, vol. 72, no. 1, pp. 26-31, 1997.

[4] F. Ichida, Y. Hamamichi, T. Miyawaki et al., "Clinical features of isolated noncompaction of the ventricular myocardium: long-term clinical course, hemodynamic properties, and genetic background," Journal of the American College of Cardiology, vol. 34, no. 1, pp. 233-240, 1999.

[5] E. N. Oechslin, C. H. Attenhofer Jost, J. R. Rojas, P. A. Kaufmann, and R. Jenni, "Long-term follow-up of 34 adults with isolated left ventricular noncompaction: a distinct cardiomyopathy with poor prognosis," Journal of the American College of Cardiology, vol. 36, no. 2, pp. 493-500, 2000.

[6] D. Sedmera, T. Pexieder, M. Vuillemin, R. P. Thompson, and R. H. Anderson, "Developmental patterning of the myocardium," Anatomical Record, vol. 258, no. 4, pp. 319-337, 2000.

[7] B. Begoña, R. Brugada, J. Brugada, and P. Brugada, "Brugada syndrome," Progress in Cardiovascular Diseases, vol. 51, pp. 122, 2008.

[8] C. Antzelevitch, P. Brugada, M. Borggrefe et al., "Brugada syndrome: report of the second consensus conference: endorsed by the Heart Rhythm Society and the European Heart Rhythm Association," Circulation, vol. 111, pp. 659-670, 2005.

[9] P. Brugada and J. Brugada, "Right bundle branch block, persistent ST segment elevation and sudden cardiac death: a distinct clinical and electrocardiographic syndrome. A multicenter report," Journal of the American College of Cardiology, vol. 20, no. 6, pp. 1391-1396, 1992.

[10] B. Martini, A. Nava, G. Thiene et al., "Ventricular fibrillation without apparent heart disease: description of six cases," American Heart Journal, vol. 118, no. 6, pp. 1203-1209, 1989.

[11] B. Martini, A. Nava, B. Canciani, and G. Thiene, "Right bundle branch block, persistent ST segment elevation and sudden cardiac death," Journal of the American College of Cardiology, vol. 22, no. 2, p. 633, 1993.

[12] S. W. Carveth, D. Olson, and J. Bechtel, "Emergency medical care system. Lincoln (Neb) mobile heart team," Archives of Surgery, vol. 108, no. 4, pp. 528-530, 1974. 
[13] T. M. Olson, V. V. Michels, J. D. Ballew et al., "Sodium channel mutations and susceptibility to heart failure and atrial fibrillation," Journal of the American Medical Association, vol. 293, no. 4, pp. 447-454, 2005.

[14] A. Frustaci, S. G. Priori, M. Pieroni et al., "Cardiac histological substrate in patients with clinical phenotype of Brugada syndrome," Circulation, vol. 112, no. 24, pp. 3680-3687, 2005. 


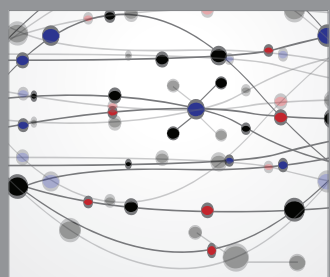

The Scientific World Journal
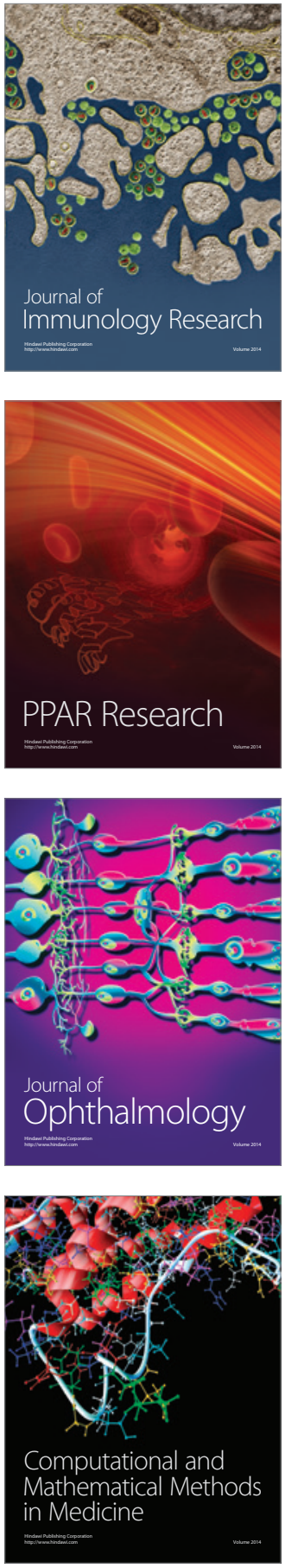

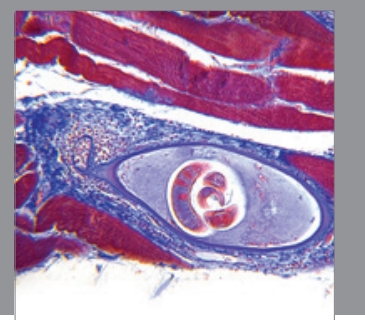

Gastroenterology

Research and Practice
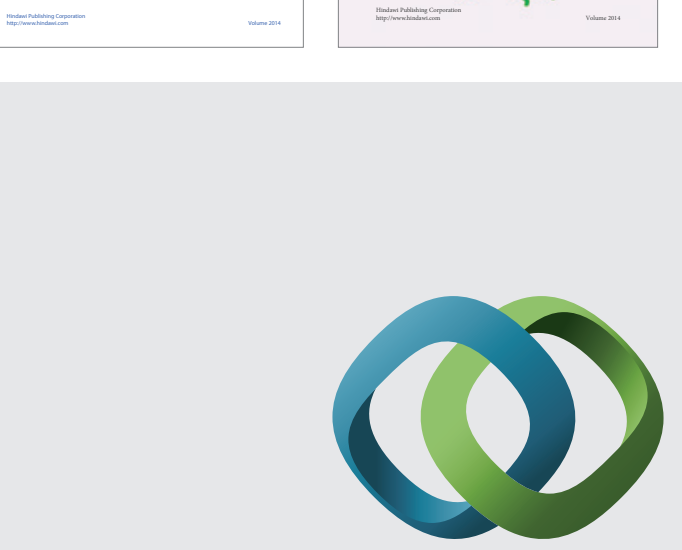

\section{Hindawi}

Submit your manuscripts at

http://www.hindawi.com
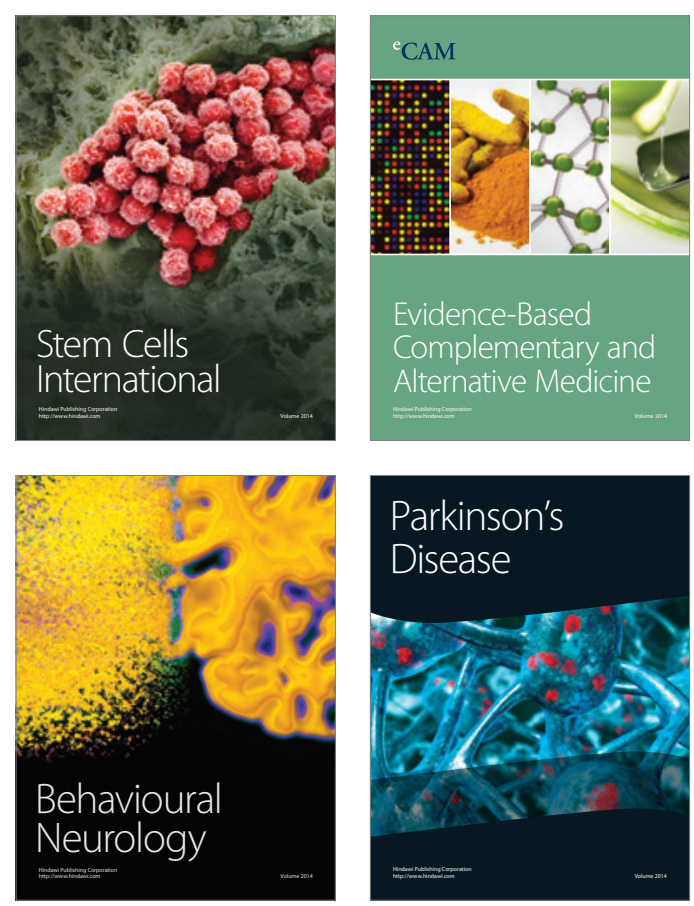

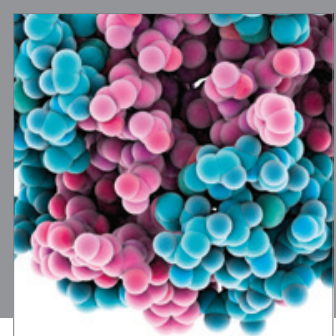

Journal of
Diabetes Research

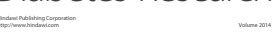

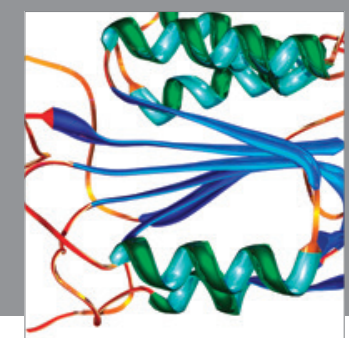

Disease Markers
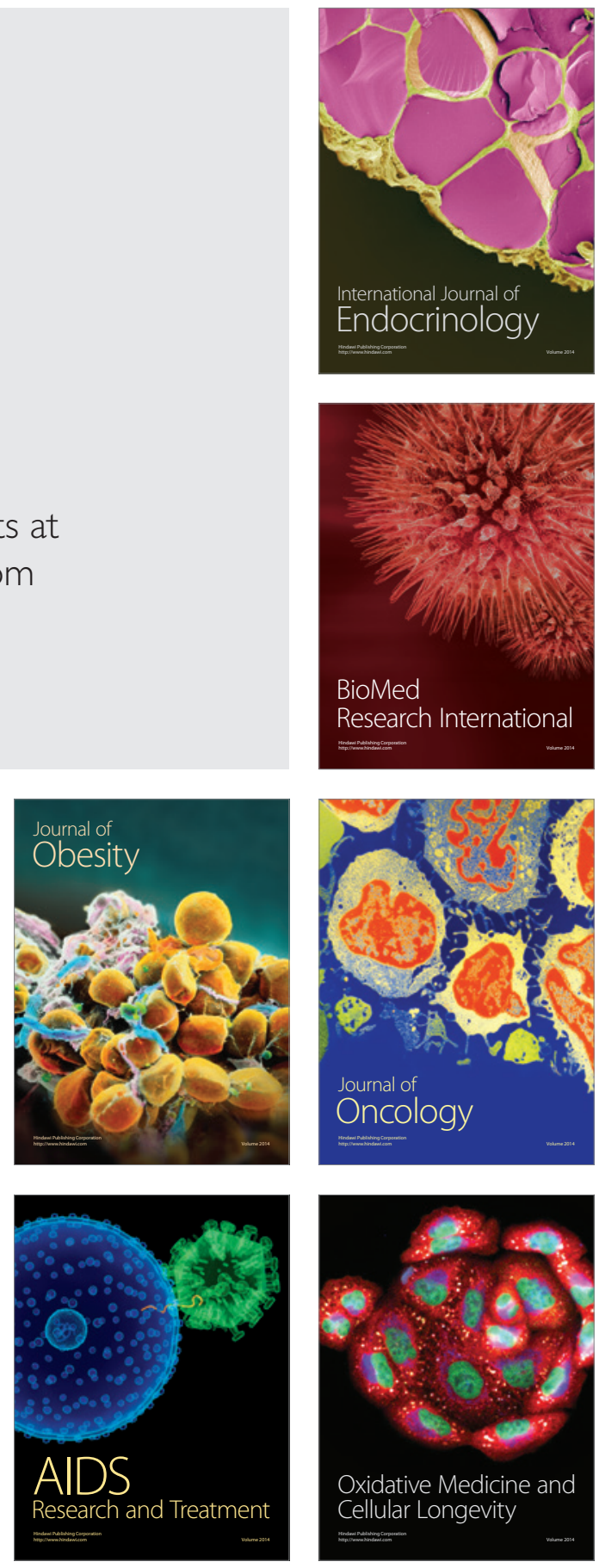